\title{
Decrease Properties of Truncated Correlation Functions and Analyticity Properties for Classical Lattices and Continuous Systems
}

\author{
M. Duneau \\ Centre de Physique Théorique, Ecole Polytechnique, Paris \\ D. Iagolnitzer
}

Service de Physique Théorique, Centre d'Etudes Nucléaires de Saclay, Gif-sur-Yvette, France

B. Souillard

Centre de Physique Théorique, Ecole Polytechnique, Paris, France

Received January 2, 1973

\begin{abstract}
We present and discuss some physical hypotheses on the decrease of truncated correlation functions and we show that they imply the analyticity of the thermodynamic limits of the pressure and of all correlation functions with respect to the reciprocal temperature $\beta$ and the magnetic field $h$ (or the chemical potential $\mu$ ) at all (real) points $\left(\beta_{0}, h_{0}\right)$ (or $\left.\left(\beta_{0}, \mu_{0}\right)\right)$ where they are supposed to hold. A decrease close to our hypotheses is derived in certain particular situations at the end.
\end{abstract}

\section{Introduction}

In a recent work [1], Lebowitz considers a ferromagnetic Ising spin lattice and presents a method which, starting from decrease properties of the correlations, proved in certain cases, allows him to derive regularity properties of the infinite-volume free energy $\psi(\beta, h)$ and correlations $\left\langle\sigma_{A}\right\rangle(\beta, h)$, at real points $\left(\beta_{0}, h_{0}\right)$ where other methods have given so far no information (e.g. at points close to $\beta_{c}$ ). However, even for finite range potentials, only infinite differentiability, rather than analyticity has been obtained. The purpose of the present paper is to present and discuss certain "physically reasonable" hypotheses, not proved in general so far, on the decrease of the truncated functions ${ }^{1}$ and to show that they do yield analyticity.

\footnotetext{
${ }^{1}$ Also called "cluster functions" or "Ursell functions" of the correlations in statistical mechanics.
} 
The hypotheses are described in Part I. In Section A, we recall the general type of decrease, called below "weak decrease", obtained and used in [1] and we see that a stronger decrease might be expected, following the idea that the truncated functions correspond to processes or correlations "involving all the particles jointly". We outline the mathematical reasons why the "weak decrease properties" do not yield analyticity in Section B. In Section C, we then present our hypotheses, called "strong decrease properties"; we indicate in Section D how they will yield analyticity, in contrast to the "weak properties" and we see that they are completely consistent with all known results, (in particular those by Ruelle [2] on the integrals of truncated functions at low activity).

In Section E, we finally describe an extension, whose usefulness will appear in Parts II, III of the hypotheses to functions which are truncated only with respect to various clusters of points.

In Parts II, III, we respectively consider classical lattices and continuous systems. In Section II.A (resp. III.A) we present general formulae expressing the successive derivatives of the pressure and of the correlation functions with respect to $\beta$ and $h$, (resp. $\beta$ and $\mu$ ), in terms of (generalized) truncated functions, and in II.B, (resp. III.B), we prove that our hypotheses do imply analyticity. In the continuous case, the truncation does not refer in general to the correlation functions $\varrho(X)$ themselves, but to related quantities which generalize the functions $\varrho(X)$ of [3] and which are introduced at the beginning of Part III.

In Part IV, we consider lattice systems with finite range interactions and we show that, whenever there is a gap in the spectrum of the transfer matrix, the $n$-point truncated correlations have a decrease of the type $e^{-\chi D}$ where $D$ is the diameter of the configuration $X=\left(x_{1}, \ldots, x_{n}\right)$ $\left(D=\operatorname{Max}_{i, j=1, \ldots, n}\left|x_{i}-x_{j}\right|\right)$, and $\chi$ is independent of $n$ (and of the configuration). This decrease is better than the "weak decrease", which, for instance in the case of equidistant points on a line, provides only a factor $e^{-\chi D / n}$. It coincides with the "strong decrease" whenever the points are lined up, but is weaker in the other situations. Concerning the problem of analyticity, the replacement of $e^{-\chi D / n}$ by $e^{-\chi D}$ is already an improvement (see footnote 2 in Part I.B). However, this is not yet sufficient to derive analyticity.

\section{Physical Hypotheses on the Truncated Functions}

\section{A. Decrease Properties of the Truncated Functions}

We consider factorization properties of the following type for the (non-truncated) correlation functions $f(X)\left(X=\left(x_{1} \ldots x_{n}\right)\right)$, when the 
distances between several clusters $X_{1} \ldots X_{M}$ of points tend to infinity:

$$
\left\{\begin{aligned}
f\left(X_{1}, X_{2}\right)-f\left(X_{1}\right) f\left(X_{2}\right) \simeq \mathcal{O}\left[u\left(d\left(X_{1}, X_{2}\right)\right)\right], \\
f\left(X_{1}, \ldots, X_{M}\right)-\prod_{j=1}^{M} f\left(X_{j}\right) \simeq \mathcal{O}\left[u\left(\operatorname{Inf}_{\substack{i, j=1, \ldots, M \\
i \neq j}} d\left(X_{i}, X_{j}\right)\right)\right]
\end{aligned}\right.
$$

where $u$ is a monotonous decreasing function vanishing at infinity, and $d\left(X_{1}, X_{2}\right)=\operatorname{Inf}\left\{d\left(x_{i}, x_{j}\right)\right\}\left(x_{i} \in X_{1}, x_{j} \in X_{2}\right)$. We remark that (2) is a consequence of (1) and that we cannot expect physically better than the infimum of the distances in (2).

The truncated functions, denoted below by T.F., are defined recurrently by:

$$
\left\{\begin{array}{l}
f^{T}(x)=f(x) \\
f^{T}\left(x_{1}, \ldots, x_{n}\right)=f\left(x_{1}, \ldots, x_{n}\right)-\sum_{\pi_{1} \ldots \pi_{k}} \prod_{j=1}^{k} f^{T}\left(x\left(\pi_{j}\right)\right)
\end{array}\right.
$$

where the sum $\sum_{\pi_{1} \ldots \pi_{k}}$ runs over all (non trivial, i.e. $k>1$ ) partitions of $x_{1}, \ldots, x_{n}$.

When factorization properties are satisfied, it is well known that the T.F. vanish at infinity as soon as certain clusters are separated from each other. In the present framework, one shows more precisely that (1), (2) are equivalent to the following decrease property, that we shall call "weak decrease":

$$
f^{T}\left(x_{1}, \ldots, x_{n}\right) \simeq \mathcal{O}\left(u\left(\operatorname{Max}_{X_{1}, X_{2}} d\left(X_{1}, X_{2}\right)\right)\right.
$$

where $X_{1}, X_{2}$ is any partition of $x_{1}, \ldots, x_{n}$ into two subsets.

Starting from (1) (2), it is not possible to get better than (4). But better decrease properties might be physically expected in various situations, namely a decrease taking into account the separation of all particles with respect to each other, and not only the maximum distance $\operatorname{Max} d\left(X_{1}, X_{2}\right)$. Truncated functions do verify such ideas in particle physics [4]. We note that we do not want conversely to get better, unexpected, asymptotic factorization properties than type (1), (2). The "strong decrease properties" of the T.F. introduced in Section C satisfy this requirement.

\section{B. Weak Decrease Properties and the Problem of Analyticity}

For the case of a ferromagnetic spin system, Lebowitz does obtain in [1] factorization properties of type (1), (2) in the form:

$$
\left|\left\langle\sigma_{X_{1} X_{2}}\right\rangle_{A}-\left\langle\sigma_{X_{1}}\right\rangle_{A}\left\langle\sigma_{X_{2}}\right\rangle_{A}\right|<\left|X_{1}\right|\left|X_{2}\right| u\left(d\left(X_{1}, X_{2}\right)\right)
$$


where $\Lambda$ is any finite region and where $u$ is independent of $\Lambda$, (and depends only on $\beta$ and $h$ ). The corresponding weak decrease of the T.F., of type (4) is:

$$
\left|\left\langle\sigma_{i_{1}, \ldots, i_{n}}\right\rangle_{\Lambda}^{T}\right|<C^{n-1}(n-1) ! u\left(\operatorname{Max}_{X_{1}, X_{2}} d\left(X_{1}, X_{2}\right)\right) .
$$

The relationship between the derivatives of the free energy $\psi_{A}$ and of the correlations $\left\langle\sigma_{A}\right\rangle_{A}$ with respect to $\beta, h$, and the T.F., then allows to obtain the infinite differentiability of the thermodynamic limits $\psi$ and $\left\langle\sigma_{A}\right\rangle$, if $u$ has a rapid decrease at infinity, (i.e. faster than any inverse power of the distance). The decrease of $u$ has actually been proved in certain cases to be exponential, for finite-range potentials and in appropriate domains in $\beta$ and $h$ : see $[1,5]$.

For instance, let us consider the behaviour of $\psi$ with respect to $h$. It is proved in [1] (see Part II.A) that

$$
\frac{\partial^{n} \psi_{\Lambda}}{\partial \tilde{h}^{n}}(\beta, \tilde{h})=\frac{1}{|\Lambda|} \sum_{i_{1}, \ldots, i_{n} \in A}\left\langle\sigma_{i_{1}}, \ldots, \sigma_{i_{n}}\right\rangle_{\Lambda}^{T}(\beta, \tilde{h}), \quad(\tilde{h}=\beta h)
$$

Then (6) yields bounds on $\frac{\partial^{n} \psi_{A}}{\partial \tilde{\mathrm{h}}^{n}}$ which are uniform with respect to $\Lambda$ as soon as $u(d)$ decreases at least like $d^{-n v-\varepsilon}$ at infinity, where $v$ is the space dimension. It is shown in [1] that those bounds entail the existence of the thermodynamic limit $\psi$ and of its derivatives.

The $n^{\text {th }}$ derivative of $\psi$ still possesses the same uniform bound as $\frac{\partial^{n} \psi_{A}}{\partial \tilde{h}^{n}}$. But the trouble which prevents from getting analyticity even when $u$ is rapidly or exponentially decreasing, is that the bounds, thus derived from (6), increase too fast with $n$ : in particular, the use of the weak decrease factor $u\left(\operatorname{Max}\left(d\left(X_{1}, X_{2}\right)\right)\right)$ induces terms such as $n^{v n}$ and $(n v) !^{2}$.

In our approach, we shall only use integrability properties of $u$ but we will require stronger decrease properties of the T.F. than (6).

\section{Strong Decrease Properties of the Truncated Functions}

Asymptotic Physics of the T.F.

We now want to express in a stronger way the idea that the T.F. correspond to correlations "involving all the particles jointly".

Consider for simplicity a three-point function $f^{T}(x, y, z)$ and the situation in which $x, y, z$ are separated from each other in a way such that $d(x, z) \gg d(x, y), d(y, z)$, see Fig. 1 :

${ }^{2}$ The decrease $e^{-\ell D}$ proved in Part IV allows one to remove the terms $n^{v n}$ (but not $(n v) !)$. 


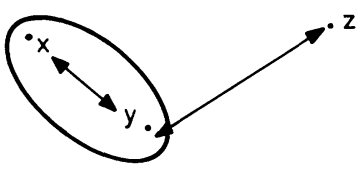

Fig. 1

We may then expect a first decrease of the order of $u(d(x, y))$ due to separation of $x$ with respect to $y$ (inside the "cluster" $x, y$ ) and a second factor of the order of $u(d(y, z))$ due to the separation of $z$ with respect to $y$. Effects due to the separation of $z$ with respect to $x$ are masked asymptotically since $u(d(z, x)) \ll u(d(x, y)), u(d(y, z))$. Therefore we are led to a decrease of the order of $u(d(x, y)) u(d(y, z))$ whereas (4) provides a decrease of the order of $u(d(y, z))$ alone.

For general $n$-point functions $f^{T}\left(x_{1}, \ldots, x_{n}\right)$, the same physical ideas lead to consider all trees $\mathscr{T}$ (i.e. connected graphs without closed loops) of $n-1$ lines joining $x_{1}, \ldots, x_{n}$ and to assume a decrease of the type:

$$
\left|f^{T}\left(x_{1}, \ldots, x_{n}\right)\right| \simeq \mathcal{O}\left(\prod_{l \in \mathscr{T}_{\min }} u\left(d_{l}\right)\right)
$$

where $\mathscr{T}_{\min }$ is the tree whose length $L$ is minimal and $d_{l}$ is the length of line $l$ (The length $\mathscr{L}(\mathscr{T})$ of a tree $\mathscr{T}$ is the sum of the lengths of its lines).

An alternative type of decrease will be presented in Section D for finite-range interactions, but will be shown to be equivalent.

The above tree $\mathscr{T}_{\min }$ can be obtained as the $n$-th term of a sequence of (connected) trees $\mathscr{T}_{p}$ joining $p$ points: $\mathscr{T}_{1}$ may be anyone of the points $x_{1}, \ldots, x_{n}$ and $\mathscr{T}_{p+1}$ is obtained from $\mathscr{T}_{p}$ by adding the line of smallest length joining a point outside $\mathscr{T}_{p}$ to a point of $\mathscr{T}_{p}$. Alternatively, $\mathscr{T}_{\text {min }}$ can be constructed by considering the set of all lines joining two points, and by successively choosing the line of smallest length, avoiding however closed loops. (These two constructions correspond to slightly different physical considerations).

\section{Bounds on the T.F}

In the following we need not only asymptotic properties, but general bounds which would extend the bound on the 2-point-function $\left\langle\sigma_{i j}\right\rangle^{T}\left(=\left\langle\sigma_{i j}\right\rangle-\left\langle\sigma_{i}\right\rangle\left\langle\sigma_{j}\right\rangle\right)$ which follows from (5) or (6).

We shall consider the assumption:

$$
\left|f^{T}\left(x_{1}, \ldots, x_{n}\right)\right|<\sum_{\mathscr{T}} \prod_{l=1}^{n-1} u\left(d_{l}\right)
$$

where the sum $\Sigma$ runs over all trees $\mathscr{T}$ (of $n-1$ lines) joining $x_{1}, \ldots, x_{n}$. 


\section{Links with Analyticity and Consistency with Known Results}

For simplicity, we here consider again the free energy $\psi$ of a spin lattice and its behaviour with respect to $h$. The function $f^{T}\left(x_{1}, \ldots, x_{n}\right)$ is here anyone of the $\left\langle\sigma_{i_{1}, \ldots, i_{n}}\right\rangle_{\Lambda}^{T}$ obtained for finite $\Lambda$; (9) will be supposed to hold in appropriate domains in $\beta$, h, and for any allowed point $\beta, h$ the corresponding function $u$ is assumed to be independent of $\Lambda$ and integrable $\left(\sum_{i \in Z^{v}} u(|i|)<\infty\right)$ : see Part II.

The contribution to the sum $\frac{1}{|\Lambda|} \sum_{i_{1}, \ldots, i_{n}}$ of (7) corresponding to a

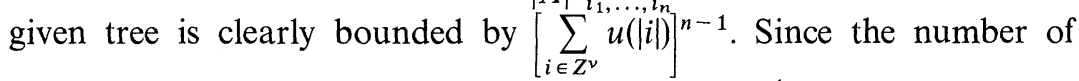
trees is itself bounded, as is easily checked, by $D^{n-1}(n-1)$ ! (where $D$ is independent of $n$ ), we get:

$$
\left|\frac{\partial^{n} \psi_{\Lambda}}{\partial \tilde{h}^{n}}\right|<\frac{1}{|\Lambda|} \sum_{i_{1}, \ldots, i_{n} \in \Lambda}\left|\left\langle\sigma_{i_{1}, \ldots, i_{n}}\right\rangle_{\Lambda}^{T}\right|<\left[D\left(\sum_{i \in Z^{\nu}} u(|i|)\right)\right]^{n-1}(n-1) !
$$

It is the use of such bounds (generalized in Parts II, III to derivatives of $\psi_{A}$ and $\left\langle\sigma_{A}\right\rangle_{A}$ with respect to $\beta$ and $h$ and to the continuous case) which will ensure the convergence of the appropriate Taylor series and will thus lead to analyticity (see Parts II and III).

\section{Consistency with Known Results}

a) Results Obtained from the Explicit Form of the T.F. [6]. 1) We below consider the example of a classical gas. The truncated correlation is then given explicitely [2] by: where

$$
\varrho^{T}\left(x_{1}, \ldots, x_{n}\right)=\sum_{N \geqq 0} \frac{z^{N+n}}{N !} \int \varphi\left(x_{1}, \ldots, x_{n}, y_{1}, \ldots, y_{N}\right) d y_{1}, \ldots, d y_{N}
$$

$$
\varphi\left(x_{1}, \ldots, y_{N}\right)=\sum_{\Gamma^{c}} \prod_{l \in \Gamma^{c}}\left(e^{-\beta \Phi\left(d_{l}\right)}-1\right) .
$$

The sum $\sum_{\Gamma^{c}}$ runs over all connected graphs joining $x_{1}, \ldots, x_{n}, y_{1}, \ldots, y_{N}$ and $d_{l}$ is the length of line $l$.

In the case of a finite-range interaction, $\Phi(d)=0$ when $d>d_{0}$, the product $\prod_{l \in \Gamma^{c}}$ vanishes whenever $d\left(l_{0}\right)>d_{0}$ for at least one line $l_{0}$ and is otherwise bounded by $e^{-\bar{\chi} N\left(\Gamma^{c}\right)}$ where $\bar{\chi}$ is positive if $\left|e^{-\beta \Phi}-1\right|<1^{3}$, and $N\left(\Gamma^{c}\right)$ is the number of lines in $\Gamma^{c}$. For given points $x_{1}, \ldots, x_{n}$, we remark that $e^{-\bar{x} N\left(\Gamma^{c}\right)}$ is always less than $e^{-\chi L^{\prime}}$ where $L^{\prime}$ is the minimal length of all (connected) trees $\mathscr{T}^{\prime}$ joining $x_{1}, \ldots, x_{n}$ and possibly other vertices.

\footnotetext{
${ }^{3}$ Or possibly equal to 1 at $d=0$.
} 
Although no such result has been proved so far, a detailed analysis of the above form of $\varrho^{T}\left(x_{1}, \ldots, x_{n}\right)$ then suggests that it might have a decrease of the type $e^{-\chi L^{\prime}}$, under appropriate conditions.

We remark that this decrease is in actual fact equivalent to (8) if we admit that the function $u(d)$ of $(8)$ is of the form $e^{-\kappa d}$ for finite-range interactions: the product $\prod_{l \in \mathscr{T}_{\min }} u\left(d_{l}\right)$ is then equal to $e^{-\kappa L}$ where $L$ is the length of $\mathscr{T}_{\min }$, and the equivalence follows from the inequalities $\frac{1}{2} L \leqq L^{\prime} \leqq L$.

The inequality $L^{\prime} \leqq L$ is obvious, since the trees $\mathscr{T}$ of Section $\mathrm{C}$ may not include other vertices than $x_{1}, \ldots, x_{n}$. To obtain the inequality $\frac{1}{2} L \leqq L^{\prime}[7]$, we note that a tree $\mathscr{T}_{0}$, whose length $\mathscr{L}\left(\mathscr{T}_{0}\right)$ satisfies $(L \leqq) \mathscr{L}\left(\mathscr{T}_{0}\right) \leqq 2 L^{\prime}$, can always be constructed from the minimal tree $\mathscr{T}_{\min }^{\prime}$ by joining directly the points $x$ which appear successively when "turning around $\mathscr{T}_{\min }^{\prime}$ ".

2) Ruelle has proved [2], at low activity and under general assumptions of stability and integrability of the potential, that the infinite-volume T.F. $\varrho_{n}^{T}\left(x_{1}, \ldots, x_{n}\right)$ are absolutely integrable with respect to the differences $x_{i}-x_{j}$. More precisely, he obtains a bound of the following type:

$$
\int\left|\varrho_{n}^{T}\left(x_{1}, \ldots, x_{n}\right)\right| d x_{2}, \ldots, d x_{n}\left\langle C^{n-1}(n-1) !\right.
$$

(where $C$ depends on $\beta$ and $\mu$ ).

The "strong decrease properties" do lead to such bounds [see for instance (10)] in contrast to the "weak decrease properties". The latter yield the infinite volume integrability of the T.F. for all $n$ if $u$ is rapidly decreasing, but the corresponding bound is even then much worse than (11) (see Section B).

A final remark: a bound better than (11) is not expected [8]. The hypotheses (9) do not indeed lead to a better bound since the number of trees is larger than $(n-1)$ !.

b) Results Obtained from the Transfer Matrix Formalism. As already mentioned, (8) has been proved in certain cases for the 2-point function $\left\langle\sigma_{i j}\right\rangle^{T}$, with $u$ being exponentially decreasing for finite-range potentials $[1,5]$. Those results will be generalized in Part IV to the $n$-point function when the points are lined up.

\section{E. Generalized Truncated Functions}

We now introduce functions $f^{T\left(X_{1}, \ldots, X_{M}\right)}(X)$, called G.T.F., which are truncated only with respect to certain subsets $X_{1}, \ldots, X_{M}$ of a partition of $x_{1}, \ldots, x_{n}$, i.e. are defined by the following formulae where 
$X_{1}, \ldots, X_{M}$ replace the individual variables $x_{1}, \ldots, x_{n}$ of (2):

$$
\begin{aligned}
f^{T(X)}(X) & =f(X) \\
f^{T\left(X_{1}, \ldots, X_{M}\right)}(X) & =f(X)-\sum_{\left(\pi_{1}, \ldots, \pi_{k}\right)}^{\left(X_{1}, \ldots, X_{M}\right)} \prod_{j=1}^{k} f^{T\left(\left\{X\left(\pi_{j}\right)\right\}\right)}\left(X\left(\pi_{j}\right)\right)
\end{aligned}
$$

where $\sum_{\pi_{1}, \ldots, \pi_{k}}^{X_{1}, \ldots, X_{M}}$ is the sum over all (non trivial) partitions of $\left(X_{1}, \ldots, X_{M}\right)$; $\left\{X\left(\pi_{j}\right)\right\}$ denotes the subsets $X_{t}$ belonging to $\pi_{j}$ and $X\left(\pi_{j}\right)$ is the set of all $x_{i}$ belonging to the various subsets of $\left\{X\left(\pi_{j}\right)\right\}$. We note that $f^{T\left(X_{1}, \ldots, X_{M}\right)}$ reduces to $f(X)$ when $M=1$, to the usual T.F. $f^{T}(X)$ when each $X_{t}(t=1, \ldots, M)$ is one point $(M=n)$, and that it can be generally expressed in terms of usual T.F. through the following formulae, which can be proved by a recurrence procedure:

$$
f^{T\left(X_{1}, \ldots, X_{M}\right)}(X)=f^{T}(X)+\sum_{\left(\pi_{1}, \ldots, \pi_{k}\right)^{c} / X_{1}, \ldots, X_{M}}^{\left(x_{1}, \ldots, x_{n}\right)} \prod_{j=1}^{k} f^{T}\left(X\left(\pi_{j}\right)\right)
$$

where the sum $\Sigma$ in the right-hand side runs over all (non trivial) partitions of $\left(x_{1}, \ldots, x_{n}\right)$ which are "connected" with respect to $X_{1}, \ldots, X_{M}$ : i.e. such that a connected diagramm of $M$ vertices is obtained when each $X_{t}$ is identified with one vertex $(t=1, \ldots, M)$ and when $X_{t_{1}}, X_{t_{2}}$ are joined by a line whenever one (or more) variable $x_{i_{1}}$ in $X_{t_{1}}$ and one (or more) variable $x_{i_{2}}$ in $X_{t_{2}}$ belong to a common $\pi_{j}(j=1, \ldots, k)$.

Strong Decrease Hypotheses for the G.T.F.

From the same physical ideas as before, we shall now assume the following "strong decrease properties" for the G.T.F, analogous to those described for the usual T.F.:

$$
\left|f^{T\left(X_{1}, \ldots, X_{M}\right)}(X)\right|<\left(\prod_{i=1}^{M} \chi\left(\left|X_{i}\right|\right)\right) \sum_{\mathscr{T}}^{\left(X_{1}, \ldots, X_{M}\right)} \prod_{l=1}^{M-1}\left(d\left(X_{l}^{\prime}, X_{l}^{\prime \prime}\right)\right)
$$

where $\sum_{\mathscr{T}}^{X_{1}, \ldots, X_{M}}$ is a sum over all trees of $M-1$ lines joining the subsets $X_{1}, \ldots, X_{M}, X_{l}^{\prime}$ and $X_{l}^{\prime \prime}$ are the subsets joined by line $l$ and $d\left(X_{l}^{\prime}, X_{l}^{\prime \prime}\right)$ is their distance.

A factor $\prod_{i=1}^{M} \chi\left(\left|X_{i}\right|\right)$ has been introduced [we shall assume that $\chi(1)=1]$. We shall not discuss it in detail from physical considerations, but we remark that a bound of type (14) is actually obtained by Lebowitz in [1] in the case of two subsets $X_{1}, X_{2}$ : see (5) [Note that $f^{T\left(X_{1}, X_{2}\right)}(X)$ $\left.=f\left(X_{1}, X_{2}\right)-f\left(X_{1}\right) f\left(X_{2}\right)\right]$. As a matter of fact, (5) in Lebowitz's proof 
is a consequence of the more refined bound:

$$
\left|\langle\sigma\rangle_{A}^{T\left(X_{1}, X_{2}\right)}\right|<\sum_{\substack{x \in X_{1} \\ y \in X_{2}}} u(d(x, y)) .
$$

A direct generalization of (15) when $M>2$, from which (14) would follow with $\chi(|X|)$ being an appropriate power of $|X|$, would be:

$$
\left|f^{T\left(X_{1}, \ldots, X_{M}\right)}(X)\right|<\sum_{\mathscr{T}}^{\left(X_{1}, \ldots, X_{M}\right)} \sum_{\substack{x_{i}^{\prime} \in X_{l}^{\prime}(\mathscr{T}) \\ x_{l}^{\prime \prime} \in X_{l}^{\prime \prime}(\mathscr{T})}} \prod_{l=1}^{M-1} u\left(d\left(x_{l}^{\prime}, x_{l}^{\prime \prime}\right)\right) .
$$

\section{A Final Remark}

We note that (9) is a particular case of (14). Conversely by using (13) the following bound may be derived from (9) for the G.T.F.:

$$
\left|f^{T\left(X_{1}, \ldots, X_{M}\right)}(X)\right|<\sum_{\Gamma^{c} / X_{1}, \ldots, X_{M}}^{x_{1}, \ldots, x_{n}} \prod_{l \in \Gamma^{c}} u\left(d\left(x_{l}^{\prime}, x_{l}^{\prime \prime}\right)\right)
$$

where the sum $\Sigma$ runs over all graphs $\Gamma^{c}$ without closed loops, which have $n$ vertices $\left(x_{1}, \ldots, x_{n}\right)$ and which are connected with respect to $X_{1}, \ldots, X_{M}^{4}$.

The bound (17) does provide the expected asymptotic behaviour of $f^{T\left(X_{1}, \ldots, X_{M}\right)}(X)$ : each term in the sum $\Sigma$ of (17) is in fact bounded by (at least) one of the terms involved in the sums $\Sigma$ of (14) (or (15)) multiplied by an appropriate power of $u(0)$. However the number of terms involved in (17) is too large for the proofs of parts II, III referring to the derivatives with respect to $\beta$. We shall then use the formulation (14) (or (15)) of our physical assumptions. Concerning the derivatives with respect to $h($ or $\mu$ ), we shall see that (17) and thus (9) are sufficient.

\section{Ferromagnetic Ising Spin Systems}

In this part, we consider classical spin lattices.

For simplicity, we shall restrict ourselves to Ising spin systems with ferromagnetic pair interactions, but the essential parts of what follows apply equally well to more general lattices. The space dimension $v$ will not be specified. In the following, we shall be interested only in cases where there is no phase transition. Therefore, the thermodynamic limits $\Lambda \rightarrow \infty$ of the correlation functions are known, from the results of [9], to be independent of the boundary conditions. For simplicity,

\footnotetext{
${ }^{4}$ The definition is here completely analogous to that given above for the connected
} partitions. 
we thus consider below free boundary conditions, but formulae analogous to those below can be written more generally.

We recall the following definitions:

$$
\begin{aligned}
H_{\Lambda} & =-1 / 2 \sum_{\substack{i, j \in \Lambda \\
i \neq j}} J_{i j} \sigma_{i} \sigma_{j}-h \sum_{i \in \Lambda} \sigma_{i}, \\
Z_{\Lambda} & =\sum_{\sigma}^{\Lambda} \exp \left(-\beta H_{\Lambda}(\sigma, h)\right), \\
\psi_{\Lambda} & =|\Lambda|^{-1} \log Z_{\Lambda}, \\
\left\langle\sigma_{A}\right\rangle_{\Lambda} & =\left(Z_{\Lambda}\right)^{-1} \sum_{\sigma}^{\Lambda} \sigma_{A} \exp \left(-\beta H_{\Lambda}(\sigma, h)\right),
\end{aligned}
$$

where $\sigma_{A}=\prod_{i \in A} \sigma_{i}$. In the following, we consider $\beta$ and $\tilde{h}=\beta h$ as independent variables.

\section{A. Derivatives of $\psi_{\Lambda}$ and $\left\langle\sigma_{A}\right\rangle_{\Lambda}$}

The following formulae [generalizing (7)] hold ${ }^{5}$ :

$$
\begin{aligned}
& \frac{\partial^{q+r} \psi_{A}}{\partial \tilde{h}^{q} \partial \beta^{r}}(\beta, \tilde{h})=|\Lambda|^{-1} \sum_{\substack{i_{1}, \ldots, i_{q} \in A \\
\gamma_{1}, \ldots, \gamma_{r} \in A^{2}}}\left(\prod_{j=1}^{r} J_{\gamma_{j}}\right)\left\langle\sigma_{i_{1}, \ldots, i_{q}, \gamma_{1}, \ldots, \gamma_{r}}\right\rangle_{A}^{T_{q}, r}(\beta, \tilde{h}), \\
& \frac{\partial^{q+r}\left\langle\sigma_{A}\right\rangle_{A}}{\partial \tilde{h}^{q} \partial \beta^{r}}(\beta, \tilde{h})=\sum_{\substack{i_{1}, \ldots, i_{q} \in A \\
\gamma_{1}, \ldots, \gamma_{r} \in A^{2}}}\left(\prod_{j=1}^{r} J_{\gamma_{j}}\right)\left\langle\sigma_{A, i_{1}, \ldots, i_{q}, \gamma_{1}, \ldots, \gamma_{r}}\right\rangle_{A}^{T_{q, r}(A)}(\beta, \tilde{h})
\end{aligned}
$$

where $T_{q, r}$ and $T_{q, r}(A)$ mean the truncation with respect to the $q$ subsets $i_{1}, \ldots, i_{q}$ of one point, the $r$ subsets $\gamma_{1}, \ldots, \gamma_{r}$ of two points and possibly the subset $A$.

When $r=0$ and $A$ is one point, (21) (22) involve only usual T.F. and are given in [1]. On the other hand, they can be extended without difficulty for $k$-body potentials $(k>2)$, the functions involved in the right-hand sides being then truncated with respect to subsets of $k$ points and less. They are readily derived, once the following formulae are proved:

$$
\begin{aligned}
\frac{\partial \psi_{\Lambda}}{\partial \tilde{h}}=|\Lambda|^{-1} \frac{1}{Z_{\Lambda}} \frac{\partial Z_{\Lambda}}{\partial \tilde{h}} & =|\Lambda|^{-1} \sum_{i \in \Lambda}\left\langle\sigma_{i}\right\rangle_{\Lambda}, \\
\frac{\partial \psi_{\Lambda}}{\partial \beta}=|\Lambda|^{-1} \frac{1}{Z_{\Lambda}} \frac{\partial Z_{\Lambda}}{\partial \beta} & =|\Lambda|^{-1} \sum_{\gamma \in \Lambda^{2}} J_{\gamma}\left\langle\sigma_{\gamma}\right\rangle_{\Lambda}, \\
\frac{\partial}{\partial \tilde{h}}\left\langle\sigma_{X}\right\rangle_{\Lambda}^{T\left(X_{1}, \ldots, X_{M}\right)} & =\sum_{i \in \Lambda}\left\langle\sigma_{X, i}\right\rangle_{\Lambda}^{T\left(X_{1}, \ldots, X_{M}, i\right)}, \\
\frac{\partial}{\partial \beta}\left\langle\sigma_{X}\right\rangle_{\Lambda}^{T\left(X_{1}, \ldots, X_{M}\right)} & =\sum_{\gamma \in \Lambda^{2}} J_{\gamma}\left\langle\sigma_{X, \gamma}\right\rangle_{\Lambda}^{T\left(X_{1}, \ldots, X_{M}, \gamma\right)} .
\end{aligned}
$$

\footnotetext{
${ }^{5}$ In the following $J_{\gamma}=0$ when $\gamma=(i, i)$.
} 
(23) (24) are easily obtained by explicit computation as well as (25) (26) when $M=1$. The general proof of (25) (26) follows by a recurrence procedure.

\section{B. Analyticity Properties of $\psi$ and $\left\langle\sigma_{A}\right\rangle$}

The existence and uniqueness of the thermodynamic limits of $\psi_{A}$ and $\left\langle\sigma_{A}\right\rangle_{A}$ are known at all points $(\beta, h)$ outside the phase transition region $\left(h=0, \beta>\beta_{c}\right)$ : see for instance [9].

We then state:

Theorem. Let $\beta_{0}, \tilde{h}_{0}$ be a (real) point at which the following bound holds uniformly for any region $\Lambda$ :

$\left|\left\langle\sigma_{A_{1}, \ldots, A_{M}}\right\rangle_{A}^{T\left(A, \ldots, A_{M}\right)}\left(\beta_{0}, \tilde{h}_{0}\right)\right|<\prod_{i=1}^{M} \chi\left(\left|A_{i}\right|\right) \times \sum_{\mathscr{T}}^{\left(A_{1}, \ldots, A_{M}\right)} \prod_{l=1}^{M-1} u\left(d\left(A_{l}^{\prime}, A_{l}^{\prime \prime}\right)\right)$

where $A_{1}, \ldots, A_{M}$ are finite subsets of $Z^{v}, u$ is integrable $\left(\sum_{i \in Z^{v}} u(|i|)<\infty\right)$ and the meaning of the right-hand side is the same as in (14).

Then the thermodynamic limits $\psi(\beta, \tilde{h})$ and $\left\langle\sigma_{A}\right\rangle(\beta, \tilde{h})$ are analytic at $\left(\beta_{0}, \tilde{h}_{0}\right)$ with respect to both variables.

Before giving the proof, we make some remarks:

1) The uniqueness of the thermodynamic limit $\left\langle\sigma_{A}\right\rangle$ which is already known from the results of [9] is also implied by a factorisation criterion on the probabilities of configurations which is due to Dobrushin [10] and which is akin to the decrease property (27) in the case of two subsets $A_{1}, A_{2}$.

2) It seems physically reasonable to assume that the bounds (27) are valid at all points $\left(\beta_{0}, \tilde{h}_{0}\right)$ outside the phase transition region. We recall that they have actually been proved by Lebowitz [1] in the case of two subsets $A_{1}, A_{2}$, under various conditions.

3) Analyticity is already known when $\tilde{h}_{0} \neq 0$ or $\tilde{h}_{0}=0$ and $\beta<\beta^{\prime}$ where $\beta^{\prime}<\beta_{c}$. (For references see for instance [11]). Thus the above theorem will give further information at all points $\tilde{h}_{0}=0$ and $\beta^{\prime} \leqq \beta_{0}<\beta_{c}$ where (27) is valid, and the assumptions of the theorem can be reduced to those points.

4) As mentioned in Part I, the analyticity of $\psi$ and $\left\langle\sigma_{A}\right\rangle$ with respect to $\tilde{h}$ alone can be derived from assumptions involving only usual T.F.

(This follows from the remarks at the end of part I and from the fact that the number of graphs $\Gamma^{c}$ is bounded by $C^{|A|+q}(|A|+q)$ ! where $C$ depends only on $|A|$, in the case of $q$ subsets of one point besides the 
subset $A$. Since $|A|$ is fixed, this factor will not modify the arguments given below).

Proof. For simplicity, we first consider $\psi(\beta, \tilde{h})$. For any finite $\Lambda, \psi_{\Lambda}$ is analytic at $\left(\beta_{0}, \hat{h}_{0}\right)$. Thus we have in a complex neighborhood of $\left(\beta_{0}, \tilde{h}_{0}\right)$ :

$$
\psi_{\Lambda}(\beta, \tilde{h})=\sum_{\substack{q \geqq 0 \\ r \geqq 0}} \frac{\left(\tilde{h}-\tilde{h}_{0}\right)^{q}\left(\beta-\beta_{0}\right)^{r}}{q !} \frac{\partial^{q+r} \psi_{\Lambda}}{\partial \tilde{h}^{q} \partial \beta^{r}}\left(\beta_{0}, \tilde{h}_{0}\right) .
$$

The successive derivatives of $\psi_{A}$ are given by (21) and the following bounds, uniform with respect to $\Lambda$, are then readily obtained from (27) in a way similar to the derivation of (10) in Part $\mathrm{I}^{6}$

$$
\left|\frac{\partial^{q+r} \psi_{\Lambda}}{\partial \tilde{h}^{q} \partial \beta^{r}}\left(\beta_{0}, \tilde{h}_{0}\right)\right| \leqq(q+r) !\left[C \sum_{i \in Z^{\nu}} u(\mid i)\right]^{q+r-1}\left[\chi(2) \sum_{i \in Z^{\nu}} J_{0 i}\right]^{r} .
$$

From (29), it follows that the series defining $\psi_{\Lambda}(\beta, \tilde{h})$ are convergent in a complex disk $D$ independent of $\Lambda$, in which a complex neighborhood of $\left(\beta_{0}, \tilde{h}_{0}\right)$ of the form $D_{1}\left(\beta_{0}\right) \times D_{2}\left(\tilde{h}_{0}\right)$ can always be included, and that the $\psi_{A}$ are moreover bounded in $D$ uniformly with respect to $\Lambda$.

For any given real $\beta_{1}$ in $D_{1}\left(\beta_{0}\right)$, [resp. any real $h_{1}$ in $D_{2}\left(\tilde{h}_{0}\right)$ ], it follows readily from Vitali's theorem [12] and from the convergence at real points that $\psi_{\Lambda}$ converges to a function $\psi$ which is analytic with respect to $\tilde{h}$, in $D_{2}\left(\tilde{h}_{0}\right)$ [resp. with respect to $\beta$, in $\left.D_{1}\left(\beta_{0}\right)\right]$. From that convergence, it follows from a second application of Vitali's theorem that for any fixed complex $\tilde{h}$ in $D_{2}\left(\tilde{h}_{0}\right)$ [resp. complex $\beta$ in $\left.D_{1}\left(\beta_{0}\right)\right] \psi=\lim \psi_{A}$ exists and is analytic with respect to $\beta$ in $D_{1}\left(\beta_{0}\right)$ [resp. $\tilde{h}$ in $\left.D_{2}\left(\tilde{h}_{0}\right)\right]$. Then Hartog's theorem [13] entails that $\psi$ is analytic with respect to both variables $\beta$ and $\tilde{h}$ in $D_{1}\left(\beta_{0}\right) \times D_{2}\left(\tilde{h}_{0}\right)$.

The analyticity of $\left\langle\sigma_{A}\right\rangle(\beta, h)$ is derived similarly.

\section{Classical Continuous Systems}

We again restrict ourselves in this part to systems of classical identical particles interacting through a two-body potential $\Phi$. As in Part II, similar results may be obtained in more general cases. We shall assume that $\Phi$ is real symmetric, stable and absolutely integrable $\left(\int_{\mathbb{R}^{v}}|\Phi(x)| d x<\infty\right)$. (Some results might however also be obtained by assuming only integrability at infinity.) For any finite region $\Lambda$ of $\mathbb{R}^{v}$, we consider the

\footnotetext{
${ }^{6}$ Use $u\left(d\left(A_{l}^{\prime}, A_{l}^{\prime \prime}\right)\right) \leqq \sum_{\substack{a_{l}^{\prime} \in A_{l}^{\prime} \\ a_{l}^{\prime \prime} \in A^{\prime \prime}}} u\left(d\left(a_{l}^{\prime}, a_{l}^{\prime \prime}\right)\right)$, and the fact that the number of products

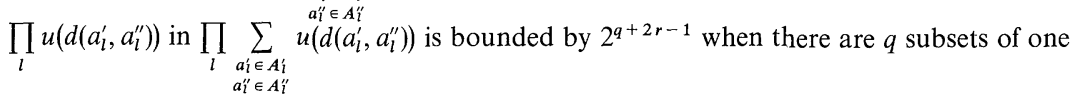
point and $r$ subsets of two points.
} 
grand canonical system for the reciprocal temperature $\beta$ and the chemical potential $\mu$. The partition function is:

$$
Z_{\Lambda}(\beta, \tilde{\mu})=\sum_{N \geqq 0} \frac{e^{N \tilde{\mu}}}{N !} \int_{\Lambda^{N}} e^{-\beta U\left(x_{1}, \ldots, x_{N}\right)} d x_{1}, \ldots, x_{N}
$$

when $\tilde{\mu}=\log z$ and $z$ is the activity.

The pressure and the correlation functions are defined (for finite $\Lambda$ ) by:

$$
\begin{gathered}
\beta p_{\Lambda}(\beta, \tilde{\mu})=|\Lambda|^{-1} \log Z_{\Lambda}(\beta, \tilde{\mu}), \\
\varrho_{\Lambda}(X)=\left(Z_{\Lambda}(\beta, \tilde{\mu})\right)^{-1} \sum_{N \geqq 0} \frac{e^{(N+n) \tilde{\mu}}}{N !} \int_{\Lambda^{N}} e^{-\beta U\left(X, Y^{N}\right)} d Y^{N} .
\end{gathered}
$$

The function $\varrho_{A}(X)$ is the "probability density" for finding $n$ different particles at points $x_{1}, \ldots, x_{n}$. In the following, quantities which appear naturally in the continuous case are functions $\varrho^{\left(X_{1}, \ldots, X_{M}\right)}(X)$ where $X_{1}, \ldots, X_{M}$ is a partition of $\left(x_{1}, \ldots, x_{n}\right)$, defined by:

$$
\varrho^{\left(X_{1}, \ldots, X_{M}\right)}(X)=\varrho(X)+\sum_{L}^{\left(X_{1}, \ldots, X_{M}\right)} \delta_{L}(X) \varrho\left(X_{L}\right)
$$

where the sum $\sum_{L}^{\left(X_{1}, \ldots, X_{M}\right)}$ runs over all "liaisons" $L$ between $X_{1}, \ldots, X_{M}$ : a liaison $L$ is composed of a certain number of "contractions", each contraction being a set of variables $x_{i}$ with at most one variable in each $X_{t}(t=1, \ldots, M)$. (A variable $x_{i}$ may not belong to more than one contraction of $L$.) With a contraction of $p$ variables is associated a product of $(p-1) \delta$-functions linking together the variables of the contraction, and $\delta_{L}(X)$ is the product of all $\delta$-functions associated with all contractions of $L$. Finally, the set $X_{L}$ is obtained from $X$ by identifying all the variables of each contraction ${ }^{7}$.

The function $\hat{\varrho}^{\left(X_{1}, \ldots, X_{M}\right)}(X)$ represents the "probability density" for finding $n_{t}=\left|X_{t}\right|$ different particles at the points of $X_{t}(t=1, \ldots, M)$, the particles belonging to different $X_{t}$ being no longer necessarily different. When each subset $X_{t}$ is reduced to one point $x_{t}, \varrho^{\left(X_{1}, \ldots, X_{M}\right)}(X)$ reduces to the function $\hat{\varrho}(X)$ introduced by Ruelle [3].

Formula (33) can be shown to be equivalent to:

$$
\begin{gathered}
\int_{\left(\Lambda^{N}\right)} \hat{\varrho}_{\Lambda}^{\left(X_{1}, \ldots, X_{M}\right)}(X) \varphi(X) d X=Z_{\Lambda}^{-1} \sum_{N \geqq 0} \frac{e^{N \tilde{\mu}}}{N !} \int e^{-\beta U\left(X^{N}\right)} d X^{N} \\
\times \sum_{J_{1}}^{\left(x_{1}, \ldots, x_{N}\right)} \ldots \sum_{J_{M}}^{\left(x_{1}, \ldots, x_{N}\right)} \varphi\left(x\left(J_{1}\right), \ldots, x\left(J_{M}\right)\right) \\
{ }^{7}\left|X_{L}\right|=|X|-\sum_{\text {contractions of } L}(p-1) .
\end{gathered}
$$


where $\varphi$ is a test function (with support in 1 ), and each sum

$\sum_{J_{t}}^{\left(x_{1}, \ldots, x_{N}\right)}(t=1, \ldots, M)$ runs over all the ordered subsets of $\left|J_{t}\right|=n_{t}\left(=\left|X_{t}\right|\right)$ variables taken from $X^{N}=\left(x_{1}, \ldots, x_{N}\right) ; x\left(J_{t}\right)$ denotes the set of $x_{j}$ in $J_{t}$. This formula is a generalization of Ruelle's formula (7.1) of [3] in which each $X_{t}$ is a point $(M=n)$.

From the functions $\hat{\varrho}^{\left(X_{1}, \ldots, X_{M}\right)}(X)$, the functions $\left(\hat{\varrho}^{\left(X_{1}, \ldots, X_{M}\right)}\right)^{T\left(X_{1}, \ldots, X_{M}\right)}$, which are truncated with respect to the same subsets $X_{1}, \ldots, X_{M}$, and are denoted below $\varrho^{\hat{T}\left(X_{1}, \ldots, X_{M}\right)}(X)$, are defined by the recurrence formulae:

$$
\varrho^{\hat{T}\left(X_{1}, \ldots, X_{M}\right)}(X)=\varrho^{\left(X_{1}, \ldots, X_{M}\right)}(X)-\sum_{\left(\pi_{1}, \ldots, \pi_{k}\right)}^{\left(X_{1}, \ldots, X_{\hat{M}}\right)} \prod_{j=1}^{k} \varrho^{\hat{T}\left(\left\{X\left(\pi_{j}\right)\right\}\right)}\left(X\left(\pi_{j}\right)\right)
$$

where the notations are the same as in (17). The functions $\varrho^{\hat{T}\left(X_{1}, \ldots, X_{M}\right)}(X)$ thus defined can be shown to possess a property analogous to (33):

$$
\varrho^{\hat{T}\left(X_{1}, \ldots, X_{M}\right)}(X)=\varrho^{T\left(X_{1}, \ldots, X_{M}\right)}(X)+\sum_{L}^{\left(X_{1}, \ldots, X_{M}\right)} \delta_{L}(X) \varrho^{T\left(\left(X_{1}, \ldots, X_{M} ; L\right\}\right)}\left(X_{L}\right)
$$

where $\left\{X_{1}, \ldots, X_{M} ; L\right\}$ is the partition of $X_{L}$ obtained by gathering all variables $x_{i}$ of any two subsets $X_{t_{1}}, X_{t_{2}}\left(t_{1}, t_{2}=1, \ldots, M\right)$ as soon as there is a contraction of $L$ joining $X_{t_{1}}$ and $X_{t_{2}}$.

\section{A. Derivatives of $\beta p_{A}$ and $\varrho_{A}(A)$}

Formulae analogous to (21) (22) hold:

$$
\begin{aligned}
& \frac{\partial^{q+r}\left(\beta p_{\Lambda}\right)}{\partial \tilde{\mu}^{q} \partial \beta^{r}}(\beta, \tilde{\mu})=\frac{(-1)^{r}}{\Lambda} \int_{\left(\Lambda^{q} \times \Lambda^{2 r}\right)} d x_{1}, \ldots, d x_{q} d Y_{1}, \ldots, d Y_{r} \prod_{j=1}^{r} \Phi\left(Y_{j}\right) \\
& \cdot e_{\Lambda}^{\hat{T}_{q}, r}\left(x_{1}, \ldots, x_{q}, Y_{1}, \ldots, Y_{r}\right) \text {, } \\
& \frac{\partial^{q+r} \varrho_{\Lambda}(A)}{\partial \tilde{\mu}^{q} \partial \beta^{r}}(\beta, \tilde{\mu})=(-1)^{r+1} \int_{\left(\Lambda^{q} \times \Lambda^{2 r}\right)} d x_{1}, \ldots, d x_{q} d Y_{1}, \ldots, d Y_{r} \prod_{j=1}^{r} \Phi\left(Y_{j}\right) \\
& \cdot \varrho_{A}^{\hat{T}_{q, r}(A)}\left(A, x_{1}, \ldots, x_{q}, Y_{1}, \ldots, Y_{r}\right)
\end{aligned}
$$

where $d Y_{j}=d u_{j} d v_{j}$ and $\Phi\left(Y_{j}\right)=\Phi\left(u_{j}-v_{j}\right)^{8}$.

As in Part II, those formulae are readily extended for $k$-body potentials, $k>2$, and are derived from:

$$
\begin{aligned}
& \frac{\partial\left(\beta p_{\Lambda}\right)}{\partial \tilde{\mu}}(\beta, \tilde{\mu})=\frac{1}{|\Lambda|} \frac{1}{Z_{\Lambda}} \frac{\partial Z_{\Lambda}}{\partial \tilde{\mu}}=\frac{1}{|\Lambda|} \int_{\Lambda} \varrho_{\Lambda}(x) d x, \\
& \frac{\partial\left(\beta p_{\Lambda}\right)}{\partial \beta}(\beta, \tilde{\mu})=\frac{1}{|\Lambda|} \frac{1}{Z_{\Lambda}} \frac{\partial Z_{\Lambda}}{\partial \beta}=-\frac{1}{|\Lambda|} \int_{\Lambda^{2}} \varrho_{\Lambda}(Y) \Phi(Y) d Y,
\end{aligned}
$$

${ }^{8} \hat{T}_{q, r}$ and $\hat{T}_{q, r}(A)$ have the same meaning as in Part II.A. 


$$
\begin{aligned}
& \frac{\partial}{\partial \tilde{\mu}} \varrho_{\Lambda}^{\hat{T}\left(X_{1}, \ldots, \hat{X}_{M}\right)}(X)=\int_{\Lambda} \varrho_{\Lambda}^{\hat{T}\left(X_{1}, \ldots, X_{M}, x\right)}(X, x) d x, \\
& \frac{\partial}{\partial \beta} \varrho_{\Lambda}^{\hat{T}\left(X_{1}, \ldots, X_{M}\right)}(X)=-\int_{\Lambda^{2}} \varrho_{\Lambda}^{\hat{T}\left(X_{1}, \ldots, X_{M}, Y\right)} \Phi(Y) d Y .
\end{aligned}
$$

(38) (39) are obtained by explicit computation. In contrast to Part II, (40) (41) are not quite trivial at $M=1 ;(41)$ for instance is then a particular case of the following formula which is verified by using (34):

$$
\begin{aligned}
& \frac{\partial}{\partial \beta} \hat{\varrho}_{\Lambda}^{\left(X_{1}, \ldots, X_{M}\right)}(X) \\
& \quad=-\int_{\Lambda^{2}} \hat{\varrho}_{\Lambda}^{\left(X_{1}, \ldots, X_{M}, Y\right)} \Phi(Y) d Y+\varrho_{\Lambda}^{\left(X_{1}, \ldots, X_{M}\right)}(X) \int_{\Lambda^{2}} \varrho_{\Lambda}(Y) \Phi(Y) d Y .
\end{aligned}
$$

The general proofs of (40) (41) follow by a recurrence procedure as in Part II.

We finally note that the derivatives with respect to $\tilde{\mu}$ alone can also be written [14]:

$$
\begin{gathered}
\frac{\partial^{q}}{\partial z^{q}}\left(\beta p_{\Lambda}\right)=\frac{1}{|\Lambda|} \frac{1}{z^{q}} \int_{\Lambda^{q}} \varrho_{\Lambda}^{T}\left(x_{1}, \ldots, x_{q}\right) d x_{1}, \ldots, d x_{q}, \\
\frac{\partial^{q}}{\partial z^{q}}\left(\frac{\varrho_{\Lambda}(A)}{z^{|A|}}\right)=\frac{1}{z^{|A|+q}} \int_{\Lambda^{q}} \varrho_{\Lambda}^{T_{q}(A)}\left(A, x_{1}, \ldots, x_{q}\right) d x_{1}, \ldots, d x_{q}
\end{gathered}
$$

where $z$ is the activity $\left(z=e^{\tilde{\mu}}\right)$.

\section{B. Analyticity Properties}

We now state:

Theorem. Let $\left(\beta_{0}, \tilde{\mu}_{0}\right)$ be a (real) point at which:

1) the thermodynamic limits $p(\beta, \tilde{\mu})$ and $\varrho(A)(\beta, \tilde{\mu})$ of the pressure and the correlation functions exist and are uniquely defined;

2) the following bounds hold uniformly with respect to $\Lambda$ for any test function $\varphi\left(y_{1}, \ldots, y_{M}\right)$ with sufficiently small support around the origin ${ }^{9}$ :

$$
\begin{array}{r}
\left|\int_{\mathbb{R}^{M \nu}} d y_{1}, \ldots, d y_{M} \varrho_{\Lambda}^{\hat{T}\left(X_{1}, \ldots, X_{M}\right)}\left(X_{1}+y_{1}, \ldots, X_{M}+y_{M}\right) \varphi\left(y_{1}, \ldots, y_{M}\right)\right| \\
\leqq C(\varphi) \prod_{j=1}^{M} \chi\left(\left|X_{j}\right|\right) \times \sum_{\mathscr{T}}^{X_{1}, \ldots, X_{M}} \prod_{l=1}^{M-1} u\left(d\left(X_{l}^{\prime}, X_{l}^{\prime \prime}\right)\right)
\end{array}
$$

where $X_{j}+y_{j}$ is the set $X_{j}$ translated by the common vector $y_{j}, u$ is an integrable function $\left(\int_{\mathbb{R}^{v}} u(|x|) d x<\infty\right)$ and $C(\varphi)$ depends only upon $\varphi$.

\footnotetext{
${ } \varrho^{\hat{T}\left(X_{1}, \ldots, X_{M}\right)} \equiv \varrho^{\hat{T}\left(X_{1}+y_{1}, \ldots, X_{M}+y_{\hat{M}}\right)}$.
} 
Then the thermodynamic limits $p$ and $\varrho(A)$ are analytic at $\left(\beta_{0}, \tilde{\mu}_{0}\right)$ with respect to both variables.

The proof is completely analogous to that of Part $\mathrm{II}^{10}$. We finally make some remarks:

1) The uniqueness of the infinite Gibbs state is probably linked with fall-off properties of the correlation functions, as for the case of lattices (see remark about Dobrushin's criterion in Part II).

2) In contrast to Part II, the $\varrho^{\hat{T}\left(X_{1}, \ldots, X_{M}\right)}(X)$ are now distributions and thus the bounds (45) refer to integrals with some test function $\varphi\left(y_{1}, \ldots, y_{M}\right)$. Bounds of the type (45) may seem plausible (provided the support of $\varphi$ is sufficiently small) outside the phase transition region and the solid phases.

3) We do not know from physical considerations whether the bounds should apply to $\varrho^{T\left(X_{1}, \ldots, X_{M}\right)}$ or $\varrho^{\hat{T}\left(X_{1}, \ldots, X_{M}\right)}$. Thus it may be useful to remark that analyticity with respect to $\tilde{\mu}$ can also be derived from assumptions analogous to (45) but referring to $\varrho^{T\left(X_{1}, \ldots, X_{M}\right)}$ (or alternatively can be derived directly from analogous assumptions referring to the usual T.F. as in Part II). To see this, it is sufficient to use (43) (44), in place of (36) (37), in the proofs.

\section{Some Results on the Decrease of the Truncated Correlations in the Ising Model}

For simplicity we consider a $v$-dimensional Ising lattice with nearest neighbour interaction, at least in one direction $\hat{l}$, and the splitting into corresponding layers.

With any given finite width of the layers is associated the set of configurations $\sigma$ of each layer, which generates a vector space $\mathscr{H}$, and the symmetric transfer matrix $T$ defined by:

$$
T\left(\sigma, \sigma^{\prime}\right)=\exp \left\{-\beta\left[\frac{H(\sigma)}{2}+\frac{H\left(\sigma^{\prime}\right)}{2}+I\left(\sigma, \sigma^{\prime}\right)\right]\right\}
$$

where $H(\sigma)$ is the energy of the configuration $\sigma$ and $I\left(\sigma, \sigma^{\prime}\right)$ is the interaction between $\sigma$ and $\sigma^{\prime}$ on two successive layers.

We shall denote by $\lambda_{0}, \lambda_{1}, \ldots, \lambda_{j}, \ldots,\left(\lambda_{0}>\lambda_{1} \geqq \cdots \geqq \lambda_{j} \ldots\right)$ and $|0>| 1>,, \ldots, \mid j>, \ldots$ the eigenvalues and eigenvectors of $T$.

Let $A_{0}, A_{1}, \ldots, A_{n}$ be arbitrary operators defined on the layers of coordinates $l_{0}, l_{1}, \ldots, l_{n}\left(l_{0}<l_{1}<\cdots<l_{n}\right)$ along $\hat{l}$.

10 The bounds on the integrals of (36) (37) are readily obtained from (45) by choosing $\varphi$ such that $\int \varphi\left(y_{1}, \ldots, y_{M}\right) d y_{1}, \ldots, d y_{M} \neq 0$ and by remarking that the function $\prod_{j=1}^{r} \Phi\left(Y_{j}\right)$ is invariant by translations $y_{j}$ of each set $Y_{j}$. 
Theorem. In the limit of an infinite number of layers, the truncated correlation function $\left\langle A_{0} A_{1} \ldots A_{n}\right\rangle^{T}$ decreases like

$$
\left(\frac{\lambda_{1}}{\lambda_{0}}\right)^{\left(l_{n}-l_{0}\right)} \equiv\left(\frac{\lambda_{1}}{\lambda_{0}}\right)^{\left(l_{1}-l_{0}\right)} \times\left(\frac{\lambda_{1}}{\lambda_{0}}\right)^{\left(l_{2}-l_{1}\right)} \times \cdots \times\left(\frac{\lambda_{1}}{\lambda_{0}}\right)^{\left(l_{n}-l_{n-1}\right)},
$$

when the distances $l_{1}-l_{0}, l_{2}-l_{1}, \ldots, l_{n}-l_{n-1}$ tend to infinity.

Proof. It is easily shown ([15]) that:

where

$$
\left\langle A_{0} A_{1} \ldots A_{n}\right\rangle=\left\langle 0\left|\tilde{A_{0}} \tilde{A_{1}} \ldots \tilde{A_{n}}\right| 0\right\rangle
$$

and correspondingly:

$$
\tilde{A_{i}}=\left(\frac{T}{\lambda_{0}}\right)^{l_{i}} A_{i}\left(\frac{T}{\lambda_{0}}\right)^{-l_{i}}
$$

$$
\left\langle A_{0} A_{1} \ldots A_{n}\right\rangle^{T}=\left\langle 0\left|\tilde{A}_{0} \tilde{A}_{1} \ldots \tilde{A}_{n}\right| 0\right\rangle^{T} .
$$

The following lemma is readily checked at $n=1$ and is then proved by recurrence.

Lemma. Let $B_{0}, B_{1}, \ldots, B_{n}$ be arbitrary operators defined on $\mathscr{H}$; let $\mathbb{Q}=\mathbf{1}-|0\rangle\langle 0|$. Then:

$$
\left\langle 0\left|B_{0} \mathbb{Q} B_{1} \ldots \mathbb{Q} B_{n}\right| 0\right\rangle=\sum_{\left\{\pi_{1}, \ldots, \pi_{k}\right\}}^{\left(0, \ldots, n_{r} r\right.} \prod_{i=1}^{k}\left\langle 0\left|B\left(\pi_{i}\right)\right| 0\right\rangle^{T}
$$

where $\sum_{\left\{\pi_{1}, \ldots, \pi_{k}\right\}}^{(0, \ldots,)_{\Gamma}}$ runs over all partitions of $(0, \ldots, n)$ (including the trivial one) which "cover" the interval $(0, n)$, i.e. are such that the set of open intervals $\left(\alpha_{i}, \beta_{i}\right)$, where $\alpha_{i}=\operatorname{Inf} \pi_{i}, \beta_{i}=\operatorname{Sup} \pi_{i}$, is a covering of $(0, n)$.

(For the recurrence, use the operator $B_{n-1}^{\prime}=B_{n-1} . B_{n}$ and formula (13).)

By applying this lemma to $\tilde{A}_{0}, \ldots, \tilde{A}_{n}$, we get:

$$
\left\langle 0\left|\tilde{A}_{0} \tilde{A}_{1} \ldots \tilde{A}_{n}\right| 0\right\rangle^{T}=\left\langle 0\left|\tilde{A}_{0} \mathbb{Q} \tilde{A}_{1} \ldots \mathbb{Q} \tilde{A}_{n}\right| 0\right\rangle-\sum_{\substack{\left\{\pi_{1}, \ldots, \pi_{k}\right\} \\ \text { (non trivial) }}}^{(0, \ldots, \mathrm{n})_{\Gamma}} \prod_{i=1}^{k}\left\langle 0\left|\tilde{A}\left(\pi_{i}\right)\right| 0\right\rangle^{T}
$$

and the announced decrease of $\left\langle A_{0} A_{1} \ldots A_{n}\right\rangle^{T}$ is then easily obtained by recurrence, using the fact that

$$
\left\|\left(\frac{T}{\lambda_{0}}\right)^{l} \mathbb{Q}\right\|=\left(\frac{\lambda_{1}}{\lambda_{0}}\right)^{l}
$$

Theorem. The same decrease is obtained in the limit of infinite width of the layers, whenever $\frac{\lambda_{1}}{\lambda_{0}}$ is strictly less than one in this limit.

This result, which generalizes the decrease obtained for the two point function ([15]), can be extended for any finite range potential and non symmetric transfer matrices.

When $A_{0}, A_{1}, \ldots, A_{n}$ have the same localization on their respective layers $l_{0}, l_{1}, \ldots, l_{n}$, the asymptotic decrease obtained coincides with the 
strong decrease of Part I.C. [However we do not obtain the precise bound (9).]

For a general localization $x_{0}, x_{1}, \ldots, x_{n}$ of $A_{0}, A_{1}, \ldots, A_{n}$ the asymptotic decrease is, at least, of the type $e^{-\chi D}$ where $D$ is the diameter of the configuration $\left(x_{0}, x_{1}, \ldots, x_{n}\right),\left(D=\underset{i, j=0, \ldots, n}{\operatorname{Max}}\left|x_{i}-x_{j}\right|\right)$, and is thus better than the "weak decrease" of part I A, which provides essentially $e^{-\chi \frac{D}{n}}([1])$. However it is not yet sufficient to derive analyticity.

Acknowledgments. We are very grateful to Drs. J. Ginibre, J. Lascoux, J. L. Lebowitz, E. H. Lieb and D. Ruelle for most interesting discussions. (However none of them should be held responsible for our "mystical" belief in the properties of the truncated functions.)

Notes added in proof: 1 . In various cases, a factor of the type $N_{1} ! \ldots N_{p} !$ is to be included in our bounds (for physical reasons) when the points $x_{1} \ldots x_{n}$ are not all different from each other. This factor does not change the derivations of analyticity.

2. The "strong decrease properties" assumed in this paper have in actual fact been proved in some situations, as described in a following paper by the same authors.

\section{References}

1. Lebowitz, J. L.: Commun. math. Phys. 28 (4), 313-321 (1972).

2. Ruelle, D.: Statistical mechanics, rigourous results, pp. 90-92. New York: Benjamin 1969.

3. Ruelle, D.: Statistical mechanics, rigourous results, p. 100. New York: Benjamin 1969.

4. Iagolnitzer, D., Stapp, H. P.: Commun. math. Phys. 14, 15-55 (1969);

Iagolnitzer, D.: Introduction to $S$-matrix theory (to be published).

5. Fisher, M. E.: Physical Rev. 162, 475 (1967).

Camp, W. J., Fisher, M.E.: Phys. Rev. B, August 1, (1972).

Marinaro, M., Sewell, G. L.: Commun. math. Phys. 24, 310 (1972).

Minlos, R.A., Sinai, J. G.: Investigations of the spectra of some stochastic operators arising in the lattice gas models. Teor. i Matem. Fizika 2 (2), 230 (1970) (russian).

6. We owe the ideas of this subsection to D. Ruelle.

7. We owe this elegant proof to C. Billionnet and C. DeCalan.

8. Arguments about this point have been given to us by D. Ruelle.

9. Lebowitz, J.L., Martin-Löf, A.: Commun. math. Phys. 25, 276 (1972).

10. Dobrushin, R.I.: Gibbsian random fields for lattice systems with pairwise interactions. Funktsional'. Analiz i Ego Prilozhen, 2 (4), 31 (1968) (russian).

11. Ruelle,D.: Statistical mechanics, rigourous results. New York: Benjamin 1969.

12. For Vitali's theorem see for instance: Goluzin, G. M.: Geometrical theory of functions of a complex variable, p. 18. AMS, Providence, Rhode Island 1969.

13. For Hartog's theorem see for instance: Bochner, S., Martin,W.T.: Several complex variables. Princeton 1948, VII, 4, Th. 4.

14. Lebowitz, J. L., Penrose, O.: Commun. math. Phys. 11, 99 (1968).

15. See for instance Camp, W. J., Fisher, M.E.: Phys. Rev. B, 6 (2), 946 (1972) where references to the transfer matrix formalism will be found.

M. Duneau

B. Souillard

Centre de Physique Théorique

Ecole Polytechnique

17 rue Descartes

F-75005 Paris, France
D. Iagolnitzer

Service de Physique Théorique

Centre d'Etudes Nucléaires de Saclay

B.P. $n^{0} 2$

F-91190 Gif-sur-Yvette, France 